\title{
Using altmetrics for assessing research impact in the humanities
}

\author{
Björn Hammarfelt ${ }^{1,2}$ \\ lbjorn.hammarfelt@abm.uu.se \\ Department of ALM, Uppsala University, Sweden \\ 2 bjorn.hammarfelt@hb.se \\ Swedish School of Library and Information Science (SSLIS), University of Borås, Sweden
}

\begin{abstract}
The prospects of altmetrics are especially encouraging for research fields in the humanities that currently are difficult to study using established bibliometric methods. Yet, little is known about the altmetric impact of research fields in the humanities. Consequently, this paper analyses the altmetric coverage and impact of humanities-oriented articles and books published by Swedish universities during 2012. Some of the most common altmetric sources are examined using a sample of 310 journal articles and 54 books. Mendeley has the highest coverage of journal articles (61\%) followed by Twitter $(21 \%)$ while very few of the publications are mentioned in blogs or on Facebook. Books, on the other hand, are quite often tweeted while both Mendeley's and the novel data source Library Thing's coverage is low. Many of the problems of applying bibliometrics to the humanities are also relevant for altmetric approaches; the importance of non-journal publications, the reliance on print as well the limited coverage of non-English language publications. However, the continuing development and diversification of methods suggests that altmetrics could evolve into a valuable tool for assessing research in the humanities.
\end{abstract}

Keywords: Altmetrics, Bibliometrics, Humanities, Mendeley, Twitter, Library Thing

\section{Introduction}

The characteristics of scholarship in the humanities have limited the application of customary bibliometric methods. Reservations against the use of these methods concern the mixed audience of research in the humanities including an international scholarly audience, a national audience as well as a public audience (Nederhof 2006). The diverse publication channels used by scholars in the humanities-articles, book chapters and monographs - are another explanation for the difficulties of applying bibliometric methods to these fields, as leading citation databases, such as Thomson Reuters Web of Science (WoS) and Elsevier Scopus, foremost index articles in English language journals. The intellectual organization of research in the humanities is yet another aspect limiting the application of bibliometrics in these fields (Hammarfelt 2012). Thus, alternative approaches for measuring impact in the humanities have been proposed due to the inadequacy of conventional methods (see for example Koshua and Thelwall 2009; Linmans 2010). This paper considers a further possible alternative to traditional bibliometric measures; altmetrics. It examines how these new 'metrics' can be used to study the humanities, and it asks if altmetrics is a viable solution for measuring the impact of research in these fields.

Altmetrics - alternative metrics usually based on data from the social web-could be 
seen as a particularly promising approach in efforts to find appropriate measures for assessing research in the social sciences and the humanities (Tang, et al. 2012). Recent studies have focused on disciplinary differences when it comes to using social media such as Mendeley (Mohammadi and Thelwall 2013), and Twitter (Holmberg and Thelwall 2013). These analyses have contributed to a further understanding of how disciplinary cultures affect the adoption of social media. A focus on data sources is common to all these studies (Mendeley or Twitter), while the actual coverage of altmetric services for specific fields is a secondary issue. The current emphasis on data sources and the possibilities they offer is warranted due to the novelty of altmetric methods. However, if altmetrics can be viewed as a possible complement or even alternative to traditional measures then the potential of altmetrics must be examined from the perspective of the overall coverage of publications. Furthermore, it is necessary to study specific areas of research as "[...] an important aspect of the evaluation of altmetrics is to identify contexts in which it is reasonable to use them." (Sud and Thellwall 2014, p. 7). Consequently, this paper analyses the altmetric coverage and 'impact' of humanities-oriented articles and books published by Swedish universities in 2012. In doing so this is one of the first studies using the total output of publications in order to examine the actual coverage of altmetric data.

The paper starts with an introduction to the growing field of altmetric research with an emphasis on disciplinary differences. It then continues with a conceptual analysis of the possibilities offered by altmetric methods and the four 'promises' of altmetrics as laid out by Wouters and Costas (2012) are reviewed. The methods and data sources used for collecting altmetric data are then introduced and the findings of the study are presented. The concluding discussion contrasts the 'promises' of altmetrics against actual findings, and implications for future research are outlined.

\section{Background}

Rapid changes in how research is disseminated have not only challenged established models for publishing but also brought into question current methods for measuring scholarly impact. Measures derived from other sources than commercial citation indices such as Web of Science or Scopus have been advocated. These new, 'altmetric' measures, propose not only to solve problems with current approaches, but they also allow for the measurement of impact beyond citations in scholarly journals. Thus, altmetrics considers all the stages and products of scholarly research from "[...] social literature search via Facebook to discussion of published results via Twitter, including any impact a publication or author may have on other people [...] (Bar-Ilan, et al. 2012, p. 2). Altmetrics is not only a growing research area but also a 'movement' with a manifesto (Priem et al. 2010), and a developing market for commercial companies offering altmetric data to researchers and institutions. Altmetrics meets many of the challenges faced by traditional bibliometrics and several researchers point to data inconsistencies as a critical issue (Priem, Piwowar and Hemminger 2012; Wouters and Costas 2012). However, the transparency of many altmetric data sources may, at least partly, be a solution to the problem of data quality (Rasmussen and Andersen 2013).

\section{Altmetric and disciplinary differences}

Disciplinary differences in referencing practices and citation patterns is a common topic in the bibliometric literature but until recently little research has been carried out on the 
influence that research practices in different fields have on the application of altmetrics. Altmetric data are often derived from social media sites such as Facebook and Twitter or social references managers like Mendeley and Cite U Like. The application of these measures is therefore dependent on the use of social media sites and services. The adoption of social media varies across research fields and a study by Rowlands and colleagues (2011) found that natural scientists were most inclined to use social media in their work. However, they also foresee that the social sciences and humanities are likely to increase their use in the near future.

One of the most studied sources used for altmetric analysis is Twitter. Holmberg and Thelwall (2013) examined the adoption of this service across disciplines finding that only $2.2 \%$ of all tweets made by researchers in the selected fields link to academic articles. Hence, they reached the conclusion that "[...] Twitter is for many researchers an important tool in scholarly communication, but it is not frequently used to share information about scientific publications." (Holmberg and Thelwall 2013, p. 578). With regard to disciplinary differences they found that scholars in the history of science were less likely to use Twitter for scholarly purposes compared with other fields. They also found that scholars in digital humanities use Twitter for facilitating conversations to quite a large degree, while researchers in the field of economics are more prone to share links. These findings are of particular interest as they correspond with earlier studies showing how references in the humanities are often used in conversational and negotiating manners (Hellqvist 2010).

The social reference manager Mendeley is another common provider of altmetric data. With over 2 million users Mendeley is a key source of altmetric data and a correlation between Mendeley readership and citation counts has been found $(\mathrm{Li}$, Thelwall and Giustini 2012). Mohammadi and Thelwall (2013) analysed the readership statistics of Mendeley for twelve disciplines using a dataset consisting of articles indexed in WoS. They found that $58 \%$ of articles from the social sciences were covered by the database while only $28 \%$ of humanities articles were indexed in Mendeley. Literature had the lowest coverage of the studied fields while library and information science had the highest. The correlation between Mendeley readership and citation counts was found to be positive for all included fields, however the correlation was stronger in fields closer to the 'hard sciences' and lower in more humanities oriented fields (Mohammadi and Thelwall 2013, p. 208).

\section{Four 'promises' of altmetric research}

Currently, a lot of attention is being paid to how altmetrics can be used to study, and eventually evaluate, the impact of scholarly publications. Advocates of this new approach to measuring the impact of research claim that altmetrics have many benefits compared to conventional bibliometric methods. In their review of the literature on the topic Wouters and Costas (2012) identified four arguments in favour of alternative metrics. These include; the diversity of dissemination channels analysed, the speed of acquiring/retrieving data, the openness of methods, and the ability to measure impact beyond the 'scholarly realm'. Below these four 'promises' are scrutinized with focus on their significance for the humanities.

\section{Diversity}


Altmetrics facilitate the analyses of many different kinds of materials. The 'impact' and visibility of scholarly journals, books, as well as blogs or 'tweets', can be studied using data available on the (social) web. The range of altmetric methods is promising, not least for many research fields in the humanities, as it opens up possibilities to measure impact beyond English language journals indexed in citation databases. Thus, the humanities with its diverse audience consisting of national and international scholars, as well as a large public audience, should benefit from an approach that encompasses many different dissemination channels.

\section{Speed}

Usually it takes considerable time to collect citation data-sometimes between two and five years - as publications have to gather citations for analysis. In addition, the time it takes for a publication to get cited is often longer in many fields in the humanities, and it has been suggested that lengthier citation windows should be used in these fields (Nederhof 2006). However, altmetric data (such as tweets or Mendeley counts) are instantly available and accessible for analysis. The use of altmetric methods could therefore be a viable solution in fields where it takes a substantial time for publications to gather citations.

\section{Openness of methods}

Altmetric data are often readily available for any researchers to download and use. In contrast with citation databases such as WoS or Scopus where an expensive license is needed in order to access the material do many providers also permit data to be reused and integrated in services like Altmetrics.com or Impact Story. The availability of data makes it possible for larger groups of researchers (including scholars in the humanities) to access metrics on their own 'impact' as well as that of others. However, as pointed out by Wouters and Costas (2012), many of the services used for altmetric analyses are only partly open, as we know very little about the inner workings of commercial companies such as Twitter or Mendeley.

\section{Beyond scholarly impact}

Altmetric methods are not restricted to the judgement of scientific authors and therefore have the potential, according to Kurtz and Bollen (2010), to cover four different groups of readers of scholarly publications - researchers, professionals, undergraduates and the interested public. The prospect of measuring the public or social impact of research is encouraging for research fields, such as history or literary studies, which often target a wide audience stretching outside academia. The potential to measure 'social' impact as well as the ability to study many different dissemination channels emerge as two strong arguments for the use of altmetric methods on the humanities.

Thus, the possibilities altmetrics offer for measuring the impact of research in the humanities, the diversity of the dissemination channels studied, as well as the option to study impact beyond the scholarly realm are especially encouraging. However, the actual possibility of gathering data from services such as Mendeley, Cite U Like, and Twitter has to be tested before these 'promises' can be evaluated. Hence, in the next section the coverage of some of the most popular sources of altmetric data is analysed. 


\section{Materials and methods}

In order to get a comprehensive sample of publications $S w e P u b$ - a database containing publication data from 30 Swedish universities - was used. The database is designed as a 'search service' and the data has to be cleaned before it can be used for statistical analysis. All publications having at least one author from a Swedish university are indexed in SwePub: thus several of the included publications are co-authored with foreign researchers. Furthermore, it should be noted that the database contains publications registered by each university so full coverage of all Swedish publications is not to be expected (http://swepub.kb.se/help.jsp. Accessed: 2014-01-21). All publications registered in the database as "humanities" for the year 2012 were selected for further analysis.

The humanities' or in some cases even 'the social science and the humanities' (SSH) are often assumed to be a readily defined set of research fields. Yet, the definition of the humanities is not a clear-cut issue, and the inclusion of research fields can differ considerably (Leydesdorff, Hammarfelt and Salah 2011). The delineation used in this paper is the one adopted by SwePub that includes 25 fields (Table 1).

Table 1. Fields defined as humanities in the SwePub-database

\begin{tabular}{ll}
\hline Antiquity Studies & History of Technology \\
Art & Language studies \\
Archaeology & Literary studies \\
Architecture & Literary writing \\
Art studies & Miscellaneous humanities \\
Comparative Language and Linguistics & Music \\
Cultural Studies & Music Studies \\
Design & Philosophy \\
Ethics & Religious History \\
Ethnology & Religious Studies \\
Film Studies & Theatre \\
History & Theatre Studies \\
History of Ideas & \\
\hline
\end{tabular}

Furthermore it is important to point out that scholarly communication can differ considerably within the humanities from a journal-oriented field such as archaeology to book-oriented scholarship in literary studies. It is also likely that the degree of social media use might differ depending on the orientation of research. Hence, conclusions drawn in this paper should be evaluated with the heterogeneous nature of research in the humanities in mind, and further, more detailed studies, are needed in order to examine the use of altmetrics in specific research fields

Table 2. Type of publications and number of publications indexed in the category of 'humanities' in the SwePub database, 2012 (Accessed, 2014-01-21)

\begin{tabular}{lll} 
Type of publication & Nr. of publications & Percentage \\
\hline Book chapters & 1470 & $(31.3 \%)$ \\
Journal articles & 1452 & $(22.7 \%)$ \\
Conference paper & 753 & $(15.6 \%)$ \\
Review & 495 & $(12.3 \%)$ \\
Other Publication & 218 & $(4.3 \%)$ \\
Book & 189 & $(4.1 \%)$
\end{tabular}




\begin{tabular}{lll} 
Editorial collection & 171 & $(3.2 \%)$ \\
Doctoral Thesis & 131 & $(2.0 \%)$ \\
Report & 94 & $(1.9 \%)$ \\
Artistic work & 55 & $(1.4 \%)$ \\
Research Review & 35 & $(0.5 \%)$ \\
Editorial Proceedings & 17 & $(0.3 \%)$ \\
Licentiate Thesis & 11 & $(0.2 \%)$ \\
\hline All Publications & $\mathbf{5 0 9 1}$ &
\end{tabular}

The total number of publications indexed under the category 'humanities' for the year 2012 was 5091 (table 2). The largest percentages of these were book chapters (31\%), followed by journal articles $(23 \%)$ and conference papers $(16 \%)$. The most common type of publication,book chapters, is particularly difficult to study as references could be given both to the specific chapter and to the book as such, and altmetric data on Swedish language publications are scarce (Hammarfelt 2013). So, although the initial aim of this study was to analyse the total output of the humanities it became apparent that the study had to be limited to English language articles and English language books.

\section{Collecting data on articles}

The Altmetric Explorer, a service provided by Altmetric.com, was used to collect data on articles from various sources (http://www.altmetric.com/). The Altmetric Explorer gathers data from social media sites such as Twitter and Facebook as well as from online reference managers Mendeley and Cite U Like. Even mentions in blog posts and in prominent newspapers, such as New York Times, are counted as an indication of 'public interest'. Data from these sources are later calculated to reach an overall 'altmetric number'. The weighted, altmetric number was not employed in this study, however, the service provided by Altmetric.com facilitated the collection of data from specific services: Twitter, Mendeley, Cite U Like, blogs and Facebook. Chamberlain (2013), among others, shows that the number of tweets or Facebook posts is inconsistent across major providers as the method and time intervals for collecting data varies between services such as Altmetrics.com, Impact Story and Plum Analytics. Hence, inconsistencies associated with the gathering of data, including the lack of transparency, have to be considered when evaluating the findings of this study.

Of the 1452 journal articles 592 were peer reviewed journal articles written in English. A prerequisite for retrieving data from Altmetric.com is that the article has a Digital Object Identifier (DOI). For some articles the DOI-number was given in SwePub but it was not always accurate and, in many cases, missing. Therefore, it was necessary to individually assign DOI before the Altmetric Explorer was applied. Each of these 592 publications was manually checked and in 310 cases the DOI could be retrieved. Thus, the altmetric record could only be assessed for a very small proportion of all journal articles. The Google Scholar citation count was included for comparison purposes. ${ }^{1}$

\section{Gathering data on books}

In total 189 books published in 2012 were indexed in SwePub, and of these 54 written in English were selected for further analysis. The collection of altmetric data on books

\footnotetext{
${ }^{1}$ Altmetric data on journal articles was gathered from 2014-01-21 to 2013-01-23.
} 
was carried out manually as a DOI (digital object identifier) is needed in order to use the automated service provided by Altmetrics.com. Therefore, Mendeley, Cite U Like and Twitter were searched individually, and an additional data source, Library Thing, was introduced for gathering altmetric data. ${ }^{2}$ The social networking site Library Thing has foremost been studied as an example of social tagging. Voorbei (2012), who looked at a random sample of monographs, found that titles from the humanities were more likely to be tagged in Library Thing compared to books from the social science and natural sciences. He also noted that the coverage of English language tended to be much higher in comparison to Dutch, German or French titles. Library Thing has already been proposed as a possible source of altmetric data (Hammarfelt 2013), but this is the first study investigating its application for assessing the impact of research.

Locating publications in Mendeley, Google Scholar and Library Thing was quite straightforward while a more refined method had to be used for Twitter. The limitations of tweets (140 characters) often result in only the first half of the title being mentioned and the author is not always named. So, the 'catchy' everyday language used in titles like this one: Crazy for Wisdom: The Making of a Mad Yogin in Fifteenth-Century Tibet requires that each tweet is individually examined to disclose if it is referring to a general expression concerning the search for wisdom or to the book in question.

\section{Findings}

Altmetric coverage of articles

Mendeley had the best coverage of altmetric datasources with $61 \%$ of the articles having at least one reader (fig 1.) A little more than $20 \%$ of the articles were mentioned on Twitter, a handful of articles where mentioned on blogs, and of the 310 articles only eight had been mentioned on Facebook. On average the articles had 3.4 readers in Mendeley and they were cited 2.4 times in Google Scholar. However, as with citation scores, these distributions are highly skewed and if the most 'read' article is removed (with 151 readers) the average drops considerably (2.9 readers and 2.2 citations).

Fig 1. Coverage of English-language journal articles with DOI, 2012. $(n=310)$

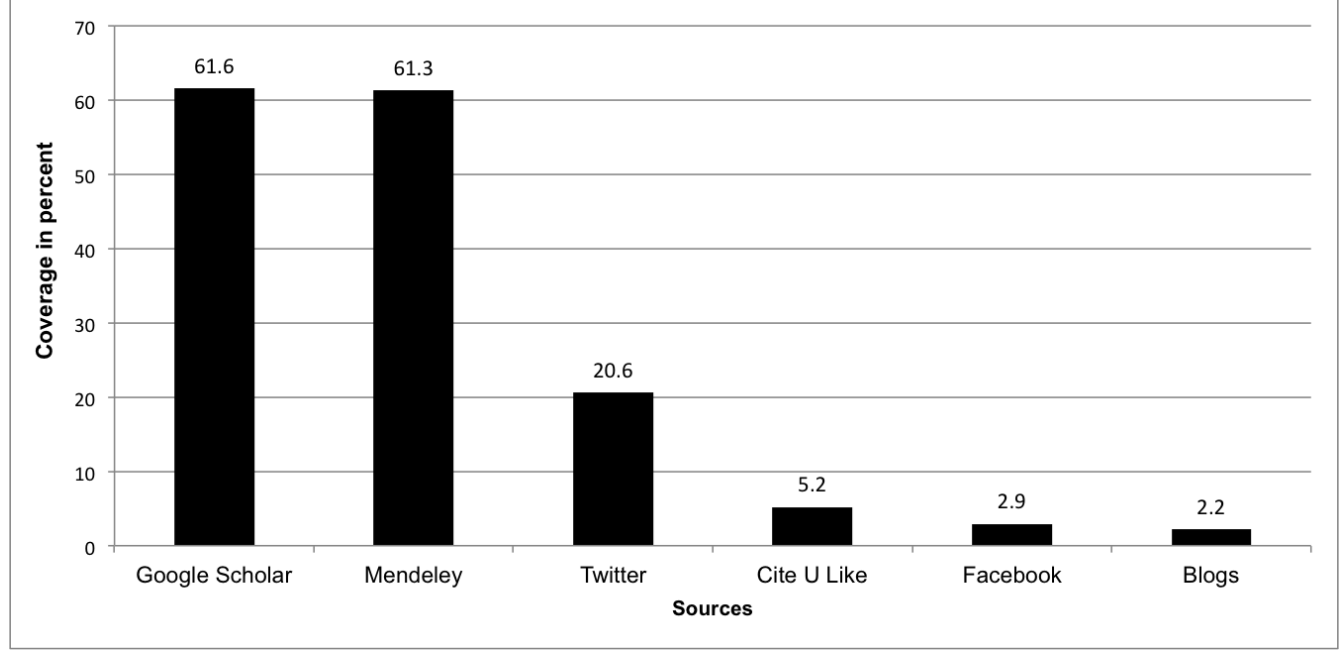

\footnotetext{
2 http://scholar.google.com/, http://www.librarything.com/search.php, https://twitter.com/search-home, http://www.mendeley.com/research-papers/search/ (Searches conducted 30-31 January 2014)
} 
Only five percent of the articles had readers in Cite $U$ Like which confirms findings from previous studies showing that Mendeley is the largest popular online reference manager and thus also one of the most useful sources for altmetric analysis ( $\mathrm{Li}$, Thellwall \& Guistini, 2011). The overall coverage is low in contrast to the field of bibliometrics and research policy where the reference database Mendeley covered $82 \%$ of the sampled outputs of researchers (Bar-Ilan et al. 2012). However, this is expected, as the research field of bibliometrics is almost exclusively oriented towards international journals that are mainly read and distributed in digital form. More surprising is the total coverage of articles (61\%), which is substantially higher than the $28 \%$ registered by Mohammadi and Thelwall (2013). One possible explanation is the small selection of research fields (history, linguistics, literature, philosophy and religion) used by Mohammadi and Thelwall (2013) while this study uses a broader definition of the humanities. Altmetric data sources, and Mendeley in particular, have a greater coverage of fields where international journal articles is the primary publication channel. Examples of journal-based fields are archaeology, linguistics and, to some extent, philosophy, here the coverage is higher when compared to book-based and more locally oriented fields such as literary studies, art or law. The different coverage for linguistics (47\%) and literature (13\%) as found by Mohammadi and Thelwall (2013) is illustrative of these differences.

To provide some insight into the properties of journal articles with a high 'altmetric score' those with 20 readers or more on Mendeley were singled out for further scrutiny (table 3). Many of the highly 'read' papers where authored by two or more authors and many of them can be considered to be interdisciplinary; at least in the sense that many of the articles are indexed in several domains crossing the humanities and the natural sciences. Journals such as Science or Health Care Analysis in which these articles are published are not perhaps intuitively indexed as 'humanities'. The inclusion of these articles is probably due to the fact that researchers themselves index their publications in SwePub, and collaborations between scholars in the humanities and social scientists or natural scientists will then be indexed in several domains.

Table 3. Journal articles having at least 20 readers on Mendeley

\begin{tabular}{|c|c|c|c|c|c|}
\hline Authors & Journal & $\begin{array}{l}\text { Mendeley } \\
\text { readers }\end{array}$ & $\begin{array}{l}\text { Twitter } \\
\text { mentions }\end{array}$ & $\begin{array}{l}\text { Google } \\
\text { Scholar } \\
\text { citations }\end{array}$ & $\begin{array}{l}\text { Field(s) } \\
*\end{array}$ \\
\hline $\begin{array}{l}\text { Skoglund, P. et } \\
\text { al. (9 authors) }\end{array}$ & Science & 151 & 53 & 55 & $\begin{array}{l}\text { HUM, } \\
\text { NAT }\end{array}$ \\
\hline $\begin{array}{l}\text { Ädel, A. \& } \\
\text { Erman, B. }\end{array}$ & English for specific purposes & 36 & 0 & 2 & HUM \\
\hline $\begin{array}{l}\text { Roos, V. et al. (6 } \\
\text { authors) }\end{array}$ & $\begin{array}{l}\text { The Science of the total } \\
\text { environment }\end{array}$ & 32 & 0 & 13 & $\begin{array}{l}\text { HUM, } \\
\text { NAT, }\end{array}$ \\
\hline $\begin{array}{l}\text { Constanza, R. et } \\
\text { al. (19 authors) }\end{array}$ & $\begin{array}{l}\text { Current Opinion in } \\
\text { Environmental Sustainability }\end{array}$ & 27 & 0 & 4 & $\begin{array}{l}\text { HUM } \\
\text { NAT }\end{array}$ \\
\hline Nordegren A. & $\begin{array}{l}\text { Journal of Agricultural and } \\
\text { Environmental Ethics }\end{array}$ & 23 & 0 & 7 & $\begin{array}{l}\text { HUM, } \\
\text { NAT }\end{array}$ \\
\hline
\end{tabular}


Welin, S.

* HUM $=$ Humanities, NAT $=$ Natural Sciences

\section{Altmetric coverage of books}

Of the 54 English-language books published by scholars at Swedish universities 25 were mentioned in tweets, 24 had one or more citations registered by Google Scholar, 14 were indexed in user 'libraries' in Library Thing and only 4 had Mendeley readers (fig 2). The low coverage of books in Mendeley is not unforeseen given the distribution of publications. A look at the statistics on indexed items in Mendeley shows that 74, 231 books are indexed compared to $15,730,970$ journal articles (Searches made on Mendeley, 2013-11-07).

Fig 2. Altmetric coverage of English-language books, 2012, $(\mathrm{n}=54)$

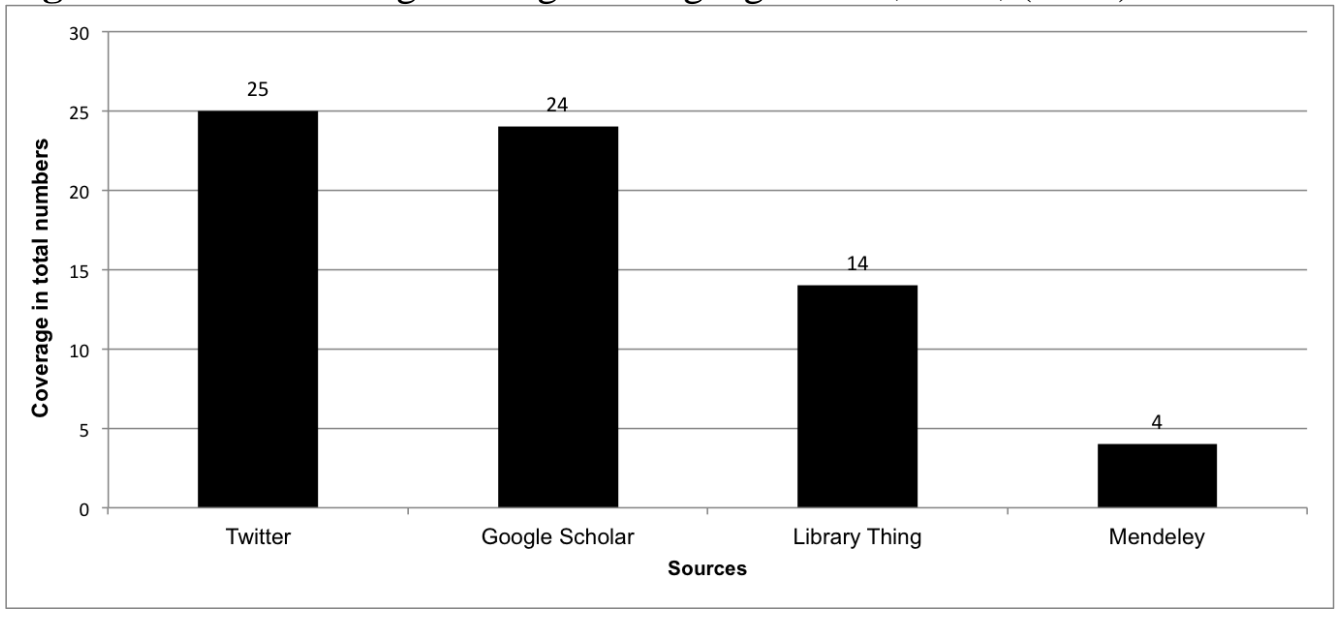

The most tweeted titled in the sample was Libraries and Archives: A Comparative Study (Chandos Publishing) with 19 tweets mostly originating from the institutions featuring in the monograph. However, despite being well-advertised on Twitter the monograph had not yet attracted any readers on Library Thing or on Mendeley, and no citations were found searching Google Scholar. From this small sample it seems that tweets advertising the book, mostly from publisher but also from the authors themselves, amounted to a considerable proportion of all tweets. For example, the book Nordic Fashion Studies was tweeted 13 times but 6 of those were tweets written by the publisher or booksellers. Thus, the number of tweets may actually be an indication of the degree of advertisement rather than impact, and it is worth highlighting that some tweets counted here were announcements of forthcoming titles posted before the book was actually published. Therefore, tweets dating from January 2011 are included in the dataset.

Overall, Mendeley stands out as the most promising altmetric source for analysing journal articles while Twitter appears to be a possible venue for assessing books. Library Thing did provide some data on books but it is doubtful if this service can be used for larger studies as only one of four books were indexed. The number of publications being blogged about, posted on Facebook or indexed in Cite U Like is negligible, and thus not useful for large and systematic analyses of publications. 


\section{Discussion}

Altmetric methods seem to solve several problems associated with the use of bibliometrics on the humanities. They allow for a multitude of sources to be analyzed, and make it possible to measure 'instant' impact in fields where citations take long time to gather. It has been argued that the variety of measures available for analysis is one of the greatest benefits of altmetrics: "Because altmetrics are themselves diverse, they're great for measuring impact in this diverse scholarly ecosystem" (Priem, et al. 2010). However, a majority of altmetric methods focus on articles in journals as the prime unit of analysis and this study illustrates how the different types of publications produced by humanities scholars contribute to the limited coverage of the humanities. Overall, it seems as though altmetric approaches work better in fields where journals are the preferred publication channel and are less effective in research fields where scholars publish in a variety of channels. However, this conclusion is not generalizable across all services providing altmetric data as Twitter actually has a larger coverage of books while the social reference manager Mendeley is better suited for analyzing the impact of articles. The proportion of articles compared to books in Mendeley substantiates this assumption; for each monograph indexed in the database there are more than 200 articles. One plausible explanation for the relatively high coverage of books in Twitter is that books target a wider audience - including an interested public. The same could be said about Twitter which is a much more general service directed to a broader audience than Mendeley's focus on academic scholarship. So, Twitter might deliver valuable insights on 'impact beyond the scholarly realm' and thus actually provide a profoundly different measure of influence compared to traditional bibliometric measures. However, findings from this study suggest that a considerable portion of all tweets mentioning books could in fact be advertisements from publishers and booksellers. Thus, we might be in danger of equating impact with promotion.

Scholarly 'ecosystems' are different in their search for and use of sources and a limitation to this study is the broad definition of the humanities. Research shows that scholars in fields such as history or literature are still more dependent on print material and library resources compared to scholars in the social sciences and natural sciences (Collins, Bulger and Meyer, 2012). This is still true even if the digitalization of books and other sources, as well as the emergence of fields such as 'digital humanities', are changing the infrastructure of research in the humanities. The continuing reliance on print has consequences for the application of altmetric methods as the frequent use of web-based social devices is often a prerequisite for analyzing impact. Hence, studies of research practices such as publishing, referencing and the use of social media are needed in the effort to develop altmetric approaches that are attuned to the 'ecology' of the humanities. A venue of research, suggested also by Sud and Thelwall (2014), that would be of great value for understanding altmetric data across disciplines is to actually look at how scholarly publications are tweeted, or blogged about (content analysis) as well as the motivation for tweeting, or adding to your library. Such studies would lay the ground for a much needed theoretical understanding of altmetric measures.

The insufficient coverage of publications in languages other than English is often seen as one of the major obstacles for evaluating the humanities using bibliometric methods. The use of bibliometric data from databases such as Web of Science is particularly worrisome in the case of major European languages such as Spanish, French, or German 
where researchers tend to focus on national audiences (Archambault et al. 2006). Altmetrics can provide a solution as the sources used are not limited to a specific database or a set of journals. The existing services, including Altmetrics.com, are foremost focused on English speaking audiences, but it is possible to imagine that the further development and refinement of altmetric methods will contribute to services that are more adapted to languages other than English, with major languages such as Chinese, French, German and Spanish being the most likely candidates for such initiatives.

The possibilities that altmetric methods offer to the humanities cannot be denied but, as shown in this paper, there are several issues that have to be addressed in order to realize their potential. Many reservations against the use of altmetric methods on the humanities relate to problems already discussed in the bibliometric literature; the diverse publication channels used by scholars in the humanities, the continuing reliance on print in many disciplines, as well as the low coverage of non-English publications. Thus, the scholarly practices of many fields in the humanities may limit the availability of altmetric data, and it would seem that current altmetric methods-with the possible exception of Twitter - share many of the limitations of traditional metrics when it come to print- and book-oriented fields. However, the digitalization and internationalization of research in the humanities, a general movement towards open access across research fields, as well as the further development and diversification of altmetric methods could, at least partly, solve the issues raised above. Then, altmetrics would be an attractive and, in many cases, superior alternative to traditional bibliometric methods for analysing and measuring the impact of research in the humanities.

\section{Acknowledgments}

Parts of this paper build on a presentation at the 14th International Conference on Scientometrics and Informetrics, Vienna, 15-19 July 2013. The author would like to thank the anonymous reviewers for insightful comments that greatly improved the article.

\section{References}

Archambault, É., Vignola-Gagne, É., Côté, G., Larivière, V., \& Gingras, Y. (2006). Benchmarking scientific output in the social sciences and humanities: The limits of existing databases. Scientometrics, 68(3), 329-342.

Bar-Ilan, J., Haustein, S., Peters, I., Priem, J., Shema, H. \& Tersliesner, J. (2012). Beyond citations: Scholars' visibility on the social Web. In E. Archambault., Y. Gingras \& V. Larivière (Eds.), Proceedings of 17th International Conference on Science and Technology Indicators, (pp. 98-109). Montréal: Science-Metrix and OST.

Chamberlain, S. (2013). Consuming Article-Level Metrics: Observations and Lessons. Information Standards Quarterly, Summer, 25(2), 4-13.

Collins, E., Bulger M. E., \& Meyer, E. T. (2012). Discipline matters: Technology use in the humanities. Arts and Humanities in Higher Education, 11(1-2), 76-92.

Hammarfelt, B. (2012). Following the Footnotes: A Bibliometric Analysis of Citation Patterns in Literary Studies. (Diss.). Uppsala: Acta Universitatis Upsaliensis.

Hammarfelt, B. (2013). An examination of the possibilities that altmetrics offer in the case of the humanities. In J. Gorraiz, E. Schiebel, C. Gumpenberger, M. Hörlesberger \& H. Moed (Eds.), Proceedings of ISSI 2013 Vienna: 14th International Society of Scientometrics and Informetrics Conference (pp. 721-727). Vienna: Austrian Institute of Technology GmbH. 
Hellqvist, B. (2010). Referencing in the humanities and its implications for citation analysis. Journal of the American Society for Information Science and Technology, 61(2), 310-318.

Holmberg, K., \& Thelwall, M. (2013). Disciplinary differences in Twitter scholarly communication. In J. Gorraiz, E. Schiebel, C. Gumpenberger, M. Hörlesberger \& H. Moed (Eds.) Proceedings of ISSI 2013 Vienna: 14th International Society of Scientometrics and Informetrics Conference (pp. 567-582). Vienna: Austrian Institute of Technology GmbH.

Kousha, K., \& Thelwall, M. (2009). Google book search: Citation analysis for social science and the humanities. Journal of the American Society for Information Science and Technology, 60(8), 1537-1549.

Kurtz, M. J., \& Bollen, J. (2010). Usage bibliometrics. Annual Review of Information Science and Technology, 44(1), 1-64.

Leydesdorff, L., Hammarfelt, B., \& Salah, A. (2011). The structure of the Arts \& Humanities Citation Index: A mapping on the basis of aggregated citations among 1,157 journals. Journal of the American Society for Information Science and Technology, 62(12), 24142426.

Li, X., Thellwall, M., \& Guistini, D. (2012). Validating online reference managers for scholarly impact measurement. Scientometrics, 91(2), 461-471.

Linmans, J. A. M. (2010). Why with bibliometrics the humanities does not need to be the weakest link. Indicators for research evaluation based on citations, library holdings and productivity measures. Scientometrics, 83(2), 337-354.

Mohammadi, E. \& Thelwall, M. (2013). Assessing the Mendeley readership of social science and humanities research. In J. Gorraiz, E. Schiebel, C. Gumpenberger, M. Hörlesberger \& H. Moed (Eds.), Proceedings of ISSI 2013 Vienna: 14th International Society of Scientometrics and Informetrics Conference (pp. 200-214). Vienna: Austrian Institute of Technology $\mathrm{GmbH}$.

Nederhof, A. J. (2006). Bibliometric monitoring of research performance in the social sciences and the humanities: A review. Scientometrics, 66(1), 81-100.

Priem, J., Piwowar, H. A., \& Hemminger, B. M. (2012). Altmetrics in the wild: Using social media to explore scholarly impact. arXiv preprint:1203.4745.

Priem, J., Taraborelli, D., Groth, P. \& Neylon, C. (2010). Altmetrics: A Manifesto. http://altmetrics.org/manifesto/. Accessed January 10, 2013.

Rasmussen, P. G., \& Andersen, J.P. (2013). Altmetrics: An alternate perspective on research evaluation. Sciecom Info, (2).

Rowlands, I., Nicholas, D., Russell, B., Canty, N., \& Watkinson A. (2011). Social media use in the research workflow. Learned Publishing, 24(3), 183-195.

Sud, P. \& Thelwall, M. (2014). Evaluating Altmetrics. Scientometrics, 98(2), 1131-1143.

Tang, M., Wang, C., Chen, K. \& Hsiang, J. (2012). Exploring alternative cyber bibliometrics for evaluation of scholarly performance in the social sciences and humanities in Taiwan. Proceedings of the ASIS\&T Annual Meeting, Vol. 49. www.asis.org/asist2012/proceedings/openpage.html. Accessed December 12, 2013.

Voorbij, H. (2012). The value of Library Thing tags for academic libraries. Online information review, 36(2), 196-217.

Wouters, P. \& Costas, R. (2012). Users, narcissism and control - Tracking the impact of scholarly publications in the 21st century. Utrecht: SURF-foundation. 